\title{
Design and Validation of a Wide Area Monitoring and Control System for Fast Frequency Response
}

\author{
Qiteng Hong, Member, IEEE, Mazaher Karimi, Member, IEEE, Mingyu Sun, Seán Norris, Member, IEEE, \\ Oleg Bagleybter, Member, IEEE, Douglas Wilson, Member, IEEE, Ibrahim Abdulhadi, Member, IEEE, \\ Vladimir Terzija, Fellow, IEEE, Ben Marshall, and Campbell Booth
}

\begin{abstract}
This paper presents the design and validation of a Wide Area Monitoring and Control (WAMC) system for Fast Frequency Response (FFR) to address the challenges associated with reduced and non-uniformly distributed inertia in power systems. The WAMC system, designed for the power system in Great Britain, is termed "Enhanced Frequency Control Capability (EFCC)". It uses real time measurements from Phasor Measurement Units (PMUs) to monitor the system state in order to rapidly detect frequency disturbances and evaluate the magnitude of power imbalances. The impact of the disturbances on different parts of the network is considered to subsequently allocate the required response for different regions of the network, all within less than one second from the initiating event. The capabilities and characteristics of different resources (e.g. wind, energy storage, demand, etc.) are also evaluated and taken into account to achieve a suitable, optimized and coordinated response. Case studies using highly realistic hardware-in-the-loop setups are presented and these demonstrate that the proposed system is capable of detecting frequency events and deploying appropriate and coordinated responses in a timely fashion even with degraded communication conditions, thereby effectively enhancing the frequency control in future low-inertia systems and permitting higher penetrations of low-carbon and low-inertia energy sources.
\end{abstract}

Index Terms-Frequency control, low inertia, PMU, wide-area monitoring and control.

\section{NOMENCLATURE}

$A \quad$ Resource availability (boolean)

$P^{+} \quad$ Available positive response

$P^{-} \quad$ Available negative response

$T_{D}^{+} \quad$ Positive response time delay

$T_{D}^{-} \quad$ Negative response time delay

$d P^{+} / d t \quad$ Positive response ramp rate

$d P^{-} / d t \quad$ Negative response ramp rate

$T_{P}^{+} \quad$ Duration of positive response

$T_{P}^{-} \quad$ Duration of negative response

$\bar{p} \quad$ Pseudo ramp rate

$f_{R_{n}} \quad$ Regional equivalent frequency in Region $n$

$f_{R_{n}}^{i} \quad$ Frequency measured by $i^{t h}$ PMU in Region $n$

$\theta_{R_{n}} \quad$ Regional equivalent angle in Region $n$

$\theta_{R_{n}}^{i} \quad$ Angle measured by $i^{\text {th }}$ PMU in Region $n$

$W_{R_{n}}^{i} \quad$ Weighting factor of $i^{t h}$ PMU in Region $n$

Q. Hong, I. Abdulhadi and C. Booth are with University of Strathclyde, UK, e-mail: q.hong@strath.ac.uk; M. Sun and V. Terzija are with University of Manchester, UK; M. Karimi is with Gonbad Kavous University, Iran. B. Marshall is with National Grid, UK. S. Norris, O. Bagleybtern and D. Wilson are with GE Power, UK.

This work is funded by National Grid, UK, under the Network Innovation Competition framework.

$\begin{array}{ll}H_{R_{n}}^{i} & \begin{array}{l}\text { Inertia constant of the } i^{t h} \text { synchronous generator } \\ \text { in Region } n\end{array} \\ S_{R_{n}}^{i} & \begin{array}{l}\text { Capacity of the } i^{t h} \text { synchronous generator in } \\ \text { Region } n\end{array} \\ Q_{R_{n}}^{i} & \text { Quality of data from of } i^{t h} \text { PMU in Region } n \\ C_{R_{n}} & \text { Confidence level of data from RA of Region } n \\ f_{S} & \text { System equivalent frequency } \\ R o C o F_{S} & \text { System equivalent rate of change of frequency } \\ \theta_{S} & \text { System equivalent angle } \\ L C_{R_{n}}^{k} & k^{t h} \text { local controller in Region } n \\ R A_{n} & \text { Regional aggregator of Region } n \\ H_{S} & \text { System equivalent inertia constant } \\ \zeta & \text { Priority value of a resource } \\ K_{R n} & \text { Impact factor of a frequency event to Region } n \\ S_{\text {rated }} & \text { Rated power of a power system } \\ \omega_{\text {syn }} & \text { Synchronous angular velocity } \\ \omega_{p u} & \text { Angular velocity in per unit } \\ \Delta P & \text { Power imbalance during a frequency event } \\ P_{R n} & \text { Required response power in region } n\end{array}$

\section{INTRODUCTION}

$\mathbf{R}$ ENEWABLE resources are typically connected to power systems via power electronics based converters, which do not naturally provide inertia to the system [1]. Therefore, the increasing penetration of renewable generation will contribute to a reduction in power system inertia. As a result, the system will become more vulnerable and responsive to frequency disturbances (e.g. loss of generation/demand due to electrical faults) - the Rate of Change of Frequency (RoCoF) will be larger and the frequency will deviate faster with the same amount of power imbalance [2]-[4]. Therefore, maintaining the frequency within acceptable limits, relying solely on conventional primary response from synchronous generators, will become increasingly challenging with a potential for significant increase in operational cost [5]. Furthermore, renewable generation is typically distributed non-uniformly in the system, which leads to variations in inertia levels and frequency behavior across different locations in the system during disturbances. Fast Frequency Response (FFR) based purely on local frequency measurement, may excite interregional power oscillations [6]. Therefore, it is critical to consider the regional impact of disturbances and dispatching resources "closest" to the event in order to ensure appropriate response and enhanced power system stability [6]-[8].

The need for FFR in low-inertia power systems has been widely recognized and FFR methods have been extensively 
investigated in recent years [9]-[15]. In [9], [10], the authors investigated the use of VSC-HVDC systems to provide fast frequency support, where control methods have been developed to make use of rotating kinetic energy within offshore windfarms and energy stored in DC links for fast frequency regulation. In [11], [12], the use of demand side response to provide FFR is reported: the authors in [11] developed a decentralized control scheme that allows the aggregation of refrigerators to provide FFR, while the work in [12] focused on demonstrating the feasibility of using a collective contribution of different types of smart loads to provide rapid frequency response. In [13], [14], [16], the authors investigated control methods that can enable DFIG-based wind turbines to provide fast active power support during frequency events. In [15], [17], [18], the authors presented new control strategies that allow PV farms to assist with frequency regulation. These publications demonstrate the importance of FFR in future power systems and the feasibility for FFR to be achieved using a range of technologies. However, the key shortcoming and gap of the existing work is that they only focus on a specific technology or type of energy source without the consideration of the optimized coordination of different resources, which could have significantly different characteristics and capabilities in providing frequency support. Furthermore, the existing work has very limited consideration of the regional impact of frequency events. In [8], the regional impact of frequency events was considered, but again only one specific resource was investigated without consideration of contribution from or coordination with other FFR providers.

The work reported in [19], [20] focuses on the optimal scheduling of resources for providing FFR. In [19], the authors present a method for allocating the commitment of energy storage to provide frequency support. In [20], the authors use a stochastic approach to schedule FFR based on the system inertia level. Again, these publications did not consider the coordination of a range of different resources and the focus is mainly on the strategy for dispatching FFRs without detailed consideration of power system's dynamic behaviors. In [21], the authors attempted to dispatch converter-interfaced resources assisted by communications to achieve FFR. However, the characteristics of resource (e.g. speed of response) are not considered, resulting in that the dispatched resources are not fully optimized to collectively provide fast and longduration response.

It can be seen from the literature review that, while the need for FFR in systems with low inertia has been widely recognized, the following issues still remain unresolved: 1) the locational impact of FFR has not been fully considered, where frequency stability has been addressed as an independent issue from rotor angle stability and the the risks of accelerating frequency control using local measurements has not been recognized; 2) the coordination of responses from different resources has not been considered when deploying the FFR from different resources with different characteristics and capability, which leads to the overall response not being optimal in performance; and 3) the need for monitoring of the status and capability of resources providing frequency response is not well recognized, which could result in the mismatch between the expected response and the actual power delivered by renewable generation.

This paper presents the design and validation of a novel Wide-Area Monitoring and Control (WAMC) system, termed "Enhanced Frequency Control Capability (EFCC)", for addressing the aforementioned frequency control challenges and the need for faster frequency response with consideration of the regional impact of events and the coordination of a range of different resources for providing the response on a localized basis. The EFCC scheme uses real time data from PMUs for monitoring the network and determining the required responses. The system takes into account of the impact of the frequency event on different parts of the network and allocates responses at a regional level. The characteristics and capabilities of different connected resources are considered, so that a fast, coordinated and optimized response is dispatched during and immediately following the event, thereby providing effective and enhanced frequency control for future power systems with low inertia.

The EFCC system presented in the paper is the first of its kind to dispatch FFR with consideration of the regional impact of frequency events using the relative magnitudes of angle separations at different regions for allocating the amount of response required in each region. This paper presents the novel design of the system architecture and the control mechanism for detecting events and deploying resources, which have never been reported and demonstrated in any existing publications. Therefore, this paper offers a timely solution to address the aforementioned frequency control challenges and minimize the risk of major frequency deviation incidents (such as the recent power cut event in the GB network [22]). Communication issues have also been fully considered in the system design to ensure the system robustness and practicality.

The paper is organized as follows: Section II describes the design of the EFCC scheme and the algorithms implemented in each of the key components; Section III presents case studies for validating the performance of the EFCC scheme using both controller and power hardware-in-the-loop arrangements, and presents the results of tests evaluating the EFCC scheme's performance when communications system performance is degraded; and the paper concludes with Section IV, which highlights the key outcomes from this work.

\section{DESIGN OF THE EFCC SCHEME FOR FAST FREQUENCY RESPONSE}

\section{A. Overview of the EFCC scheme}

The EFCC scheme has been designed with the following high-level requirements:

- Bound total response magnitude to predictably limit over/under response to events;

- Response must be within 500 ms of event initiation;

- Minimize impact of control actions on the transient stability of the network;

- Maximize utilization of available EFCC resources;

- Be capable of dealing with communications performance issues and failures in resource deployment. 


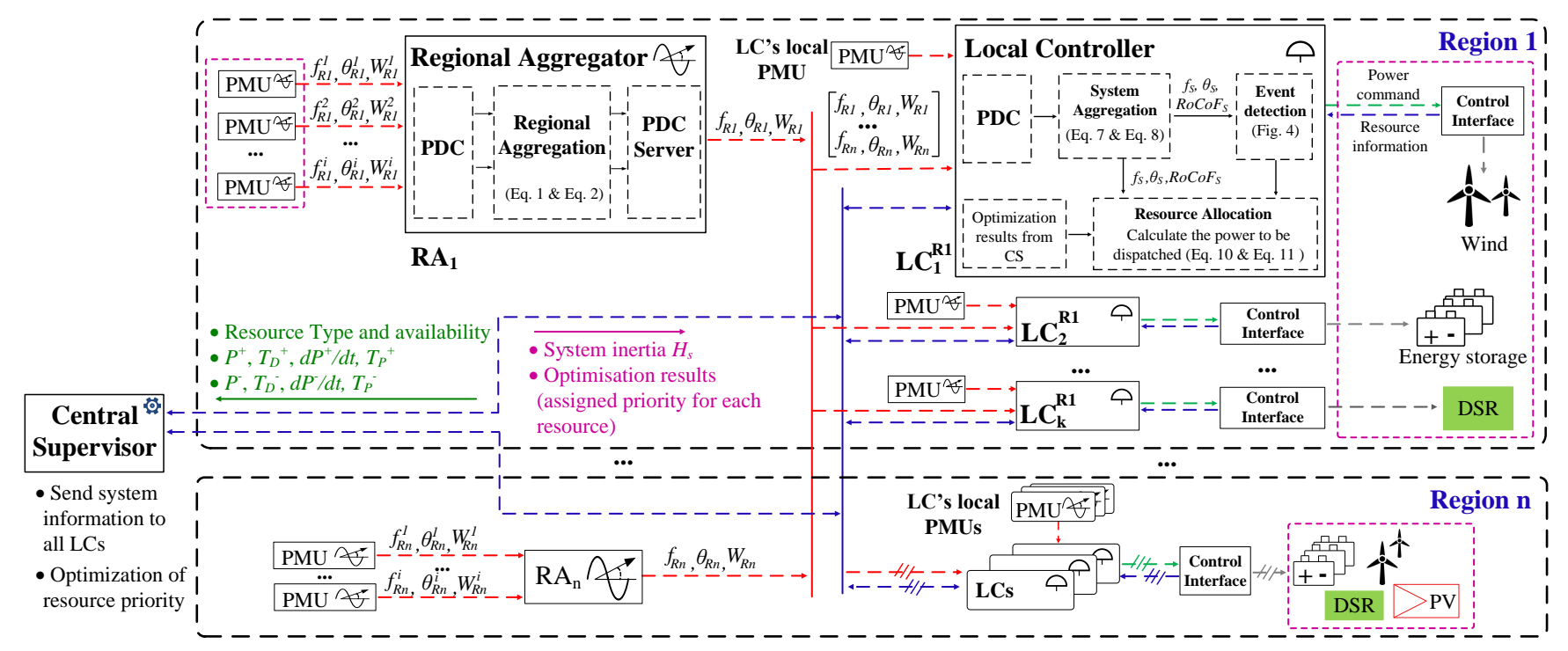

Fig. 1. Schematic of the design of the EFCC scheme

The design of the scheme is illustrated in Fig. 1. The system uses a distributed control approach as indicated in [23], where the monitoring and control functions are executed using three functional elements: the Central Supervisor (CS), Local Controllers (LCs) and Regional Aggregators (RAs).

The scheme is designed around two main data paths: one real-time "fast-communication" path to communicate grid measurements and monitoring data (illustrated with red lines in Fig. 1), which is typically sent every $20 \mathrm{~ms}$ for real-time event detection and determination of control action; and a nearreal-time communication path (blue lines in Fig. 1) for sharing resource information and the optimization results to prioritize the most effective resources (e.g. short delay, large ramp rate, etc.) to be deployed as response.

For the near-real-time data path (marked in blue), the CS uses it to gather information about the capabilities, characteristics and availability of resources that are providing the EFCC response. Since the resource availability information is not expected to change over very short timescales, such information will only be sent and updated to the CS periodically (e.g. 10s of seconds or minuets, which is configurable) or whenever there is a significant change in the resource information. An example of significant change in resource information could be where a resource becomes unavailable or the forecasted wind and PV have changed significantly resulting in the said resources not being able to provide the response they lastly reported. Based on the resource information, the CS identifies the optimal combination of resources to achieve the most desirable coordinated response, e.g. a short delay and high ramp up capability with sufficient duration. The CS sends the optimization results (in the form of a set of priority values and rankings for each resource) and system operating conditions to all LCs. This information is used by the LCs when calculating the required responses from individual resources. This is discussed in Section II-C and II-D. The optimization process does not need to be conducted in real time, and is only required when there is an update in the resource information in the system. This allows the optimization to be achieved even with a large number of resources.

For the fast-data measurements, the power system is divided into regions, where the boundaries would be formed based on suitable coherency studies [24]. Within each region, a number of PMUs are installed close to the dominant inertial sources in the region. This PMU data is collected by the RA, which contains aggregating algorithms to produce an equivalent frequency and angle value for its region. The outputs of the RAs are broadcast to all LCs, which perform real-time monitoring and control functions. The LCs are installed at the resource sites that provide the FFR service. Each resource is equipped with one LC and a local measurement device (typically a PMU), which acts as a backup in the event of loss of wide-area communications

When a disturbance occurs, each of the distributed LCs detects the event based on the wide-area measurements, or the local backup measurement. Each LC will then determine the overall response required for the event and from that, their individual contribution to the overall response. The contribution of the regions, and hence individual resources within the regions, is determines based on analysis of the wide-area phase angle movement as a result of the event. Deploying response with respect to the wide-area phase angle movement ensures minimal impact on the overall transient stability of the system. The selection of which particular resource to use within each region is based on the information received pre-event from the CS. Using the priority tables, resources are deployed according to their effectiveness in halting the frequency decline.

It should be noted that the CS only performs a coordination role for resources, ranking resources according to how effective they can be during a frequency event and it does not perform any real-time control functions. The real-time control actions are performed autonomously by the LCs, which are installed at the resource sites. Such a distributed control mechanism is a key novelty of the scheme that ensures the robustness of the overall system in the case of communication 
failures. In an extreme case, where there is a complete failure of communication system, the LCs are also equipped with a local operational mode, where they will still be able to provide fast response but using local measurement as opposed to wide area measurements. The only disadvantage of the local mode is there will be no consideration of locational impact of events or coordinated control as the wide area visibility is lost. However, this is what happens to existing schemes that use local measurements and it will only happen to the EFCC when there is a complete failure of the communication network, which is extremely unlikely. Since the local mode is not the key contribution of the work, so the paper will focus on the wide-area coordinated control of the EFCC scheme.

It should also be noted that EFCC aims to deploy responses with a shorter time delay than conventional droopbased responses, thereby enhancing containment of frequency deviations. Droop control is still required in the system for frequency regulation. The design of each of the three main elements and the algorithms to achieve the associated functions are described in detail in the following sections.

\section{B. Design of Regional Aggregators (RAs)}

An RA is installed in each region to collect and process PMU measurements and produce a regionally aggregated frequency and angle to represent the regional behavior. Compared to a centralized control scheme, this has the benefit of reducing the amount of data sent from a region. Also, it reduces the effect of local modes of oscillations in a region through the aggregating function. In order to represent a region's frequency, sufficient PMUs are required within the region to represent the inertial resources' frequency behaviors, and ensure that the effects of system reconfiguration/outage and resource unavailability do not significantly impact the accuracy of the overall measurement synthesis. A weighting factor $\left(W_{R n}^{i}\right)$ is assigned to each PMU measurement to reflect its observability of the surrounding inertia in the region. Clearly, in real-world applications, there are threats to data quality, measurement accuracy and communications systems integrity. Accordingly, quality handling is incorporated in all functions.

The weighting factors for PMUs are largely governed by their locations, where the PMUs used in the aggregation should be in locations which capture local inertia behaviour. Therefore, all large inertial sites (e.g. nuclear plants, CCGTs, etc.) should be directly monitored. By utilizing the PMUs that are connected at, or close to the larger inertia sites in the GB system, the main inertial behaviour of the system can be captured. $W_{R n}^{i}$ is defined as:

$$
W_{R_{n}}^{i}=\frac{H_{R_{n}}^{i} \times S_{R_{n}}^{i}}{\sum\left(H_{R_{n}}^{i} \times S_{R_{n}}^{i}\right)}
$$

The regional equivalent frequency is calculated using (2):

$$
f_{R n}=\frac{\sum f_{R n}^{i} \times W_{R n}^{i} \times Q_{R n}^{i}}{\sum f_{R n}^{i} \times W_{R n}^{i}}
$$

where: $Q_{R n}^{i}$ is a quality metric derived for the measurement data based on elements such as communications quality and

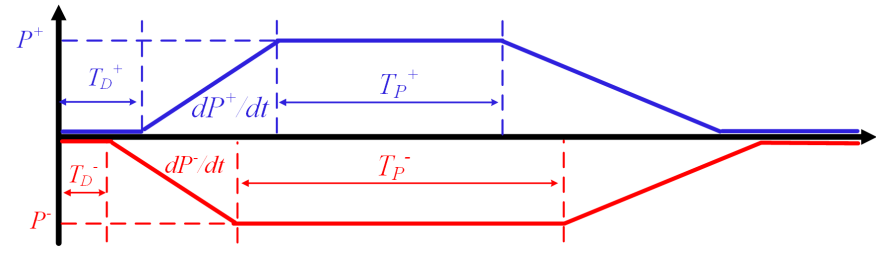

Fig. 2. Response characteristics of resources

signal metadata such as defined by the IEEE C37.118.2 standard [25].

The regional aggregated angle $\theta_{R n}$ is calculated using a similar method as shown in (3).

$$
\theta_{R n}=\frac{\sum \theta_{R n}^{i} \times W_{R n}^{i} \times Q_{R n}^{i}}{\sum \theta_{R n}^{i} \times W_{R n}^{i}}
$$

A confidence level of the region is defined to assess the quality and the availability of PMU data in the RA is calculated as shown below in (4):

$$
C_{R n}=\frac{\sum W_{R n}^{i} \times Q_{R n}^{i}}{\sum W_{R n}^{i}}
$$

In the LC, which processes the RA data, the confidence level $C_{R n}$ is used to determine if the signal from a particular region is of sufficient quality to be used by the scheme. If the confidence from that regions calculation is too low, it will be excluded from calculations, however, this does not prevent the scheme from operating. This is part of the graceful degradation design of the scheme.

The output of an RA is a vector $\boldsymbol{R}=\left[\begin{array}{lll}f_{R n} & \theta_{R n} & C_{R n}\end{array}\right]$, which will be used by LCs for detecting events and calculating the response required in each region.

\section{Design of Central Supervisor (CS)}

The main task of the CS is to inform all LCs regarding the latest system condition (e.g. overall system inertia) and provide near-real time assessment and coordination of resources across the system. Based on the availability, capabilities and characteristics of the resources that can provide FFR, an optimization process is performed by the CS to prioritize the available resources in terms of response speed and duration capabilities.

Each resource providing FFR is characterized as shown in Fig. 2 using a number of parameters to describe its capability, e.g. $P^{+}$and $d P^{+} / d t$ describe the total amount of positive power and the ramp up rate of the positive active power respectively. The LCs will report such information to the CS periodically or whenever there is a change to the resource status. The CS will receive this information from all LCs and perform the optimization function to assign resource priorities, i.e. ranking resources' frequency response effectiveness.

In this example of the optimization algorithm, the priority value of a resource $(\zeta)$ is calculated based on the speed by which a resource can respond and the duration of that response. From Fig. 2, it can be seen that, the speed of response is associated with delay and ramp rate, i.e. $T_{D}^{+}$and $d P^{+} / d t$ for positive response. Therefore, a pseudo ramp rate 


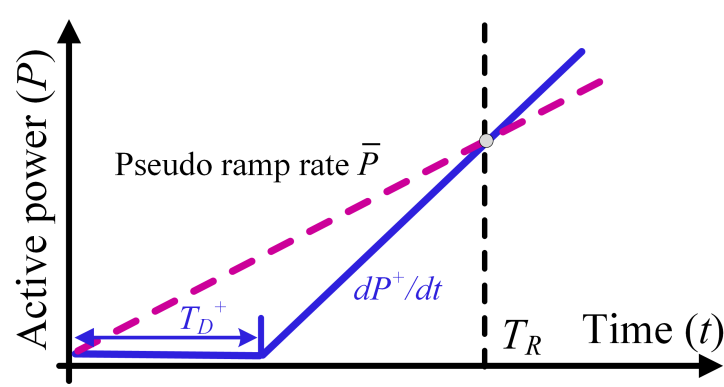

Fig. 3. Definition of the pseudo ramp rate

$(\bar{P})$, associated with delay and ramp rate, is defined to compare the response speed of different resources. The definition of pseudo ramp rates is illustrated in Fig. 3. In this section, the positive response of a resource for under-frequency event is used as the example to explain the design of CS. The negative direction of response will follow the same principle

Firstly, the resource profile can be expressed as (5):

$$
P=\frac{d P^{+}}{d t} \times T_{R}-\frac{d P^{+}}{d t} \times T_{D}^{+}
$$

At a defined time $T_{R}$, the pseudo ramp rate $\bar{P}$ can be calculated as (6):

$$
\bar{P}=\frac{\frac{d P^{+}}{d t} \times T_{R}-\frac{d P^{+}}{d t} \times T_{D}}{T_{R}}
$$

The value of $T_{R}$ is configured based on the desired response window, i.e. $1-2 \mathrm{~s}$ where it effectively represents the regional deployment of resource once triggering action has occurred, but also accounting for delays in the resource deployment.

Once the pseudo ramp rate is calculated, the priority of each resource may be obtained using (7):

$$
\zeta_{k}=\alpha \frac{\bar{P}^{k}}{\max (\bar{P})}+\beta \frac{T_{P}^{k}}{\max \left(T_{P}\right)}
$$

where $\bar{P}^{k}$ and $T_{P}^{k}$ are the pseudo ramp rate and the power duration of the $k^{t h}$ resource in a region respectively, while $\max (\bar{P})$ and $\max \left(T_{P}\right)$ are the largest ramp rate and the longest duration for all resources within a region; $\alpha$ is the weighting of positive/negative response rate; $\beta$ is the weighting of positive/negative response duration. A relatively higher priority will be assigned to a resource with a faster response and/or longer duration capability compared to other resources. The weights $\alpha$ and $\beta$ are configurable settings in the CS.

The calculated priority values $\left(\zeta_{k}\right)$ will be used to produce a ranking table for all the resources in the region. The ranking table is sent to the LCs, along with system information (e.g. system inertia, number of active regions and resources, etc.), which is used by the LCs to determine the nature and amount of their responses.

\section{Design of Local Controllers (LCs)}

The LCs are distributed controllers that are installed at (or close to) resource sites. LCs autonomously detect events and calculate the resource deployment based on real-time measurements from RAs and ranking information from the
CS. There are a number of steps and function blocks involved in the decision making process; these are described in detail in the following subsections.

1) System aggregation: The outputs from RAs contains information relating to regional behavior, such as the effects of inter-area oscillations. To evaluate the system power imbalance from an event, the data from RAs is further aggregated using the same methodology as used in the RA, producing a system-wide equivalent frequency and angle using (8) and (9) respectively. This process is referred to as system aggregation and it is necessary for: a) creating a system signal from which to detect system events; b) creating the reference by which to compare each of the regions for the locational targeting of the control scheme; and c) providing a filtering effect for inter-area oscillations due to averaging between the oscillating regions. The latter is important as it reduces the effects of inter-area oscillations on the performance of event detection and subsequent power imbalance calculations, each of which would otherwise be sensitive to the effects of these oscillations.

$$
\begin{aligned}
f_{S} & =\frac{\sum f_{R n} \times W_{R n} \times Q_{R n}}{f_{R n} \times W_{R n}} \\
\theta_{S} & =\frac{\sum \theta_{R n} \times W_{R n} \times Q_{R n}}{\theta_{R n} \times W_{R n}}
\end{aligned}
$$

2) Event detection: The control scheme is only triggered when a frequency event is detected. The effects of local phenomena such as line trips, which will affect local frequency measurements, do not trigger the scheme. The events are detected using a pre-defined RoCoF threshold. Conventional methods of calculating RoCoF from PMU measurements can lead to a long delay due to the averaging window used. For this scheme, a novel fast event detection algorithm is used, which limits the delays associated with fixed windowing methods. The approach uses a window-fitting approach to target the samples surrounding the frequency excursion. The detection window contains frequency values which are received every $20 \mathrm{~ms}$ from the measurement devices. The detector employs multiple stages of detection for verification, and best-fit calculations to increase the accuracy of the detection method without unnecessary delay. There are a series of settings which allows user configuration and tuning of the behavior of the detection algorithms, such as changing thresholds, adjusting sensitivity, etc. The algorithm is designed to "ride-through" gaps in data which may occur in wide-area networks, and remain operational when gaps are present.

The event detector algorithm will provide an event trigger but also a RoCoF calculation, where the event window method captures the event gradient more effectively than a fixed window approach as shown in Fig. 4. Use of a smaller window allows faster detection, as the calculated RoCoF will violate the triggering threshold sooner, but also provides a faster assessment of the resultant system RoCoF due to the event.

3) Resource allocation: Once an event is detected, the LC must determine the amount of individual power contribution from the resource it is controlling, based on the scale of the detected event, the impact of the event on its region and the ranking information from the CS. This process is referred to 


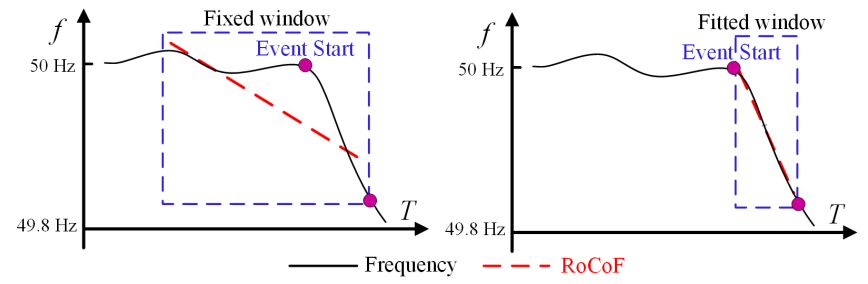

Fig. 4. Comparison of detection window on RoCoF

as resource allocation. The event scale is estimated using the swing equation as shown in (10) [26]:

$$
\frac{2 H_{S} \times S_{\text {rated }}}{\omega_{\text {syn }}} \times \omega_{p u} \times R o C o F_{S}=\Delta P
$$

$\Delta P$ is the estimated total amount of power imbalance following a frequency disturbance. The system inertia $H_{s}$ should be equivalent to the current inertia in the system, but this may be difficult to accurately assess, so the value used in the algorithm allows for error, using a self-correcting mechanism during deployment. When an event occurs, the LCs use the wide-area measurements to evaluate which regions are most affected by the disturbance, identifying the leading and lagging regions based on their individual angular acceleration.

A factor $K_{R n}$ is defined using (11):

$$
K_{R n}=\underbrace{\Delta \theta_{R n}}_{\theta_{R n}^{-}-\theta_{R n}^{+}}-\underbrace{\Delta \theta_{S}}_{\theta_{S}^{-}-\theta_{S}^{+}}
$$

where, $\theta_{S}^{-}$and $\theta_{R n}^{-}$are pre-event system and regional angle respectively, while $\theta_{S}^{+}$and $\theta_{R n}^{+}$are system and regional angle at the time when the event is detected. The impact of an event on each region can therefore be quantified through the above analysis of angles deviation levels due to the event.

The total power imbalance is then allocated to the most affected regions, but biased initially towards the regions which are "leading" the event, i.e. ahead of the system frequency. By targeting control to these regions, the angular separation between the regions can be reduced, thus reducing the risk of transient instability or system splits. The amount of power, $P_{R m}$, that a region requires to respond appropriately to the event can be calculated using (12).

$$
P_{R m}=\frac{K_{R m}}{\sum_{j=1}^{m} K_{R j}} \times \Delta P
$$

Regions $m$ represent the regions that are allowed to provide frequency response during the initial control period where the risk of instability is a concern.

Once the effects of the event on the angular separation have passed, typically after the first swing following the event, the risk of applying resources without region bias is reduced. The scheme will then transition to a coordinated control stage, where additional resources from regions which were initially blocked can be utilized, i.e. regions not included in Regions $m$ can begin to respond and contribute to the frequency response. This is particularly useful if there was insufficient resource available in the most affected regions. The chosen design

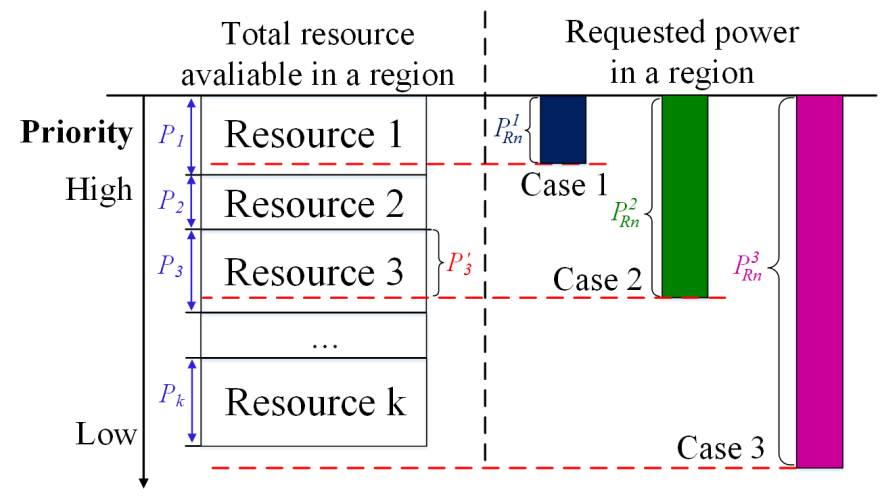

Fig. 5. Examples of resource allocation within a region

principle maximizes the use of available resources without jeopardizing system stability.

Within each region, there can be multiple resources controlled by multiple LCs. The responses from these resources are coordinated by their capability and characteristics so that the overall response is fast and of sufficient duration for handover to primary response. This is illustrated in Fig. 5. The total requested power will be taken from the available power based on the priority, which is determined by the CS based on the resources' ramp rate and duration (as presented in Section II-C). In case 1 , resource 1 is sufficient to supply all the requested power and has the highest priority, so all requested power will be dispatched from resource 1 . In case 2 , the requested power is resource 1 and 2 will be fully dispatched while the remaining power will be taken from resource 3 . If the requested power is greater than the available power, all the available power will be dispatched as shown in case 3 .

It should be noted that the EFCC scheme focuses on the application of fast acting resources for frequency response, e.g. energy storage, PV, wind, etc. These resources are typically connected to the system via convertors without dedicated governors, so the power command from the LCs will be directly used to control the active power outputs of the resources. One exceptional case is the CCGT, which is synchronous generation but is suitable for use as EFCC resources due to their relatively fast response capability than other types of synchronous generators. In this case, the power commands from the LCs will go directly to the power reference setting of the governors, which typically use droop-type control.

\section{VALIDATION OF THE EFCC SCHEME}

In this section, the methods used for testing the EFCC scheme and the associated test results are presented to demonstrate the effectiveness of EFCC in enhancing frequency control in a low-inertia system. The validation of the scheme has been performed in two main stages using ControllerHardware-in-the-Loop (C-HiL) and Power-Hardware-in-theLoop (P-HiL) approaches respectively. C-HiL and P-HiL are techniques that have been widely used for realistic testing of prototype systems [27]. C-HiL is suitable for applications where the interaction between simulation and the physical devices only involves analog and digital signals [28], while 


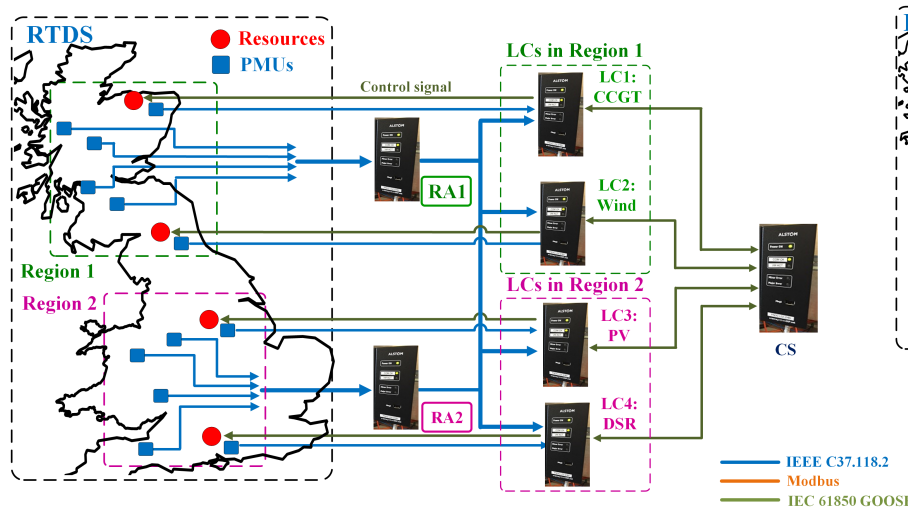

(a) Test setup for C-HiL validation of the EFCC system

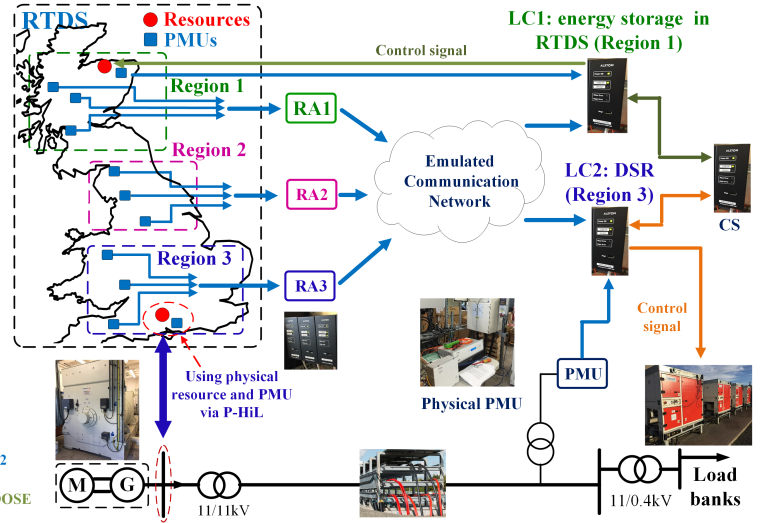

(b) Test setup for P-HiL validation of the EFCC system

Fig. 6. Test setups for validation of the EFCC scheme

P-HiL is suitable for applications where there is power interaction between the simulation and the physical system [29].

The main reason for testing the EFCC scheme using both of these two approaches is that $\mathrm{C}-\mathrm{HiL}$ is more flexible and easier in configuration of the network operating conditions, which allows a relatively large number of tests to be conducted with a lower cost and shorter period of time. For P-HiL, it involves real power devices, so it is more costly and takes longer time to run the tests. However, the P-HiL approach is more realistic to emulate the real field trial environment as it allows the EFCC to be tested with physical PMUs and power resources. Therefore, in this work, the EFCC scheme was firstly validated using C-HiL and subsequently the P-HiL approach. The EFCC control algorithms were run on industrial substation hardened, hard-real-time wide-area controllers with PLC engine [30]. The weighting factors $\alpha$ and $\beta$ as shown in (7) are set to 0.7 and 0.3 respectively, i.e. the speed of response is considered as more important than duration based on studies in [29].

\section{A. C-HiL validation of the EFCC scheme}

The C-HiL test setup for validating the EFCC scheme is illustrated in Fig. 6.(a). A reduced GB transmission network model has been constructed in RTDS for emulating frequency disturbances. The RTDS model has 26 buses and has been developed based on the model reported in [31]. In the model, there are 20 synchronous generators with IEEE governor Type 1 [32] , 25 converter-interfaced generation units and 26 lumped loads. The system is configured to have two regions based on generator coherency group studies [24]. The development of the GB network model in RTDS using the software platform RSCAD is discussed in detail in [33].

In these tests, the EFCC scheme contains four LCs, two RAs and one CS. The network is configured to have two different regions (corresponding to the two RAs), with each region containing a mix of synchronous generators and converter-based generation. There are in total four resources (corresponding to the four LCs), with each region containing two resources providing the EFCC responses. The resource information is provided in Table I. The network model is dispatched to represent the lowest inertia level with stored kinetic energy of 82 GVAs in the GB transmission system in 2021/22 based on the studies conducted in [2]. The frequency profiles with and without EFCC, for the same system disturbance, are compared to demonstrate the benefit of incorporating the fast response from EFCC. It should be noted that when the system is without EFCC, the response is provided solely by conventional governor-based controllers to contain frequency, which will be demonstrated to be insufficient in a low-inertia system.

In the first test (Case A1), a loss of $1 \mathrm{GW}$ generation event was triggered in Region 2, where LC3 and LC4 are located. As shown in Fig. 7.(a), The event occurred at $4.76 \mathrm{~s}$, and was successfully detected at $5.00 \mathrm{~s}$. LC3 and LC4, locating in the same region as the event, deployed full amount of power immediately when the event was detected at $5.00 \mathrm{~s}$. The frequency nadir is improved from less than $49.36 \mathrm{~Hz}$ to 49.67 $\mathrm{Hz}$ with EFCC scheme. After a few seconds of the event, LC1 has issued a small amount of response to further compensate the power deficit in the system.

In the second test (Case A2), the location of the $1 \mathrm{GW}$ event is moved to Region 1, where there is only $200 \mathrm{MW}$ from LC1CCGT. As shown in Fig. 7.(b), similar to the previous case, the event occurred at $4.76 \mathrm{~s}$ and detected at $5.00 \mathrm{~s}$. LC1 deployed all of its resource, and the frequency nadir is improved from less than $49.36 \mathrm{~Hz}$ to $49.45 \mathrm{~Hz}$. However, in this case, due to LC2 is not available, the response from LC1 do not appear to be sufficient compared to previous case in region 2. Therefore, LC3-PV has issued additional response after a few seconds when system transient has settled down.

\section{B. P-HiL validation of EFCC scheme}

The P-HiL testbed for validating the EFCC scheme is shown in Fig. 6.(b). The testbed contains two main parts: a reduced GB transmission network model simulated in RTDS and an 11 $\mathrm{kV}$ physical network with physical load banks. The network model is a similar model as used in the C-HiL, but has been configured to have 3 regions for the purpose of more diverse testing. The simulated network model is coupled with the 11 $\mathrm{kV}$ physical network through P-HiL synchronization using a MW scale motor-generator set. Effectively, this setup allows 

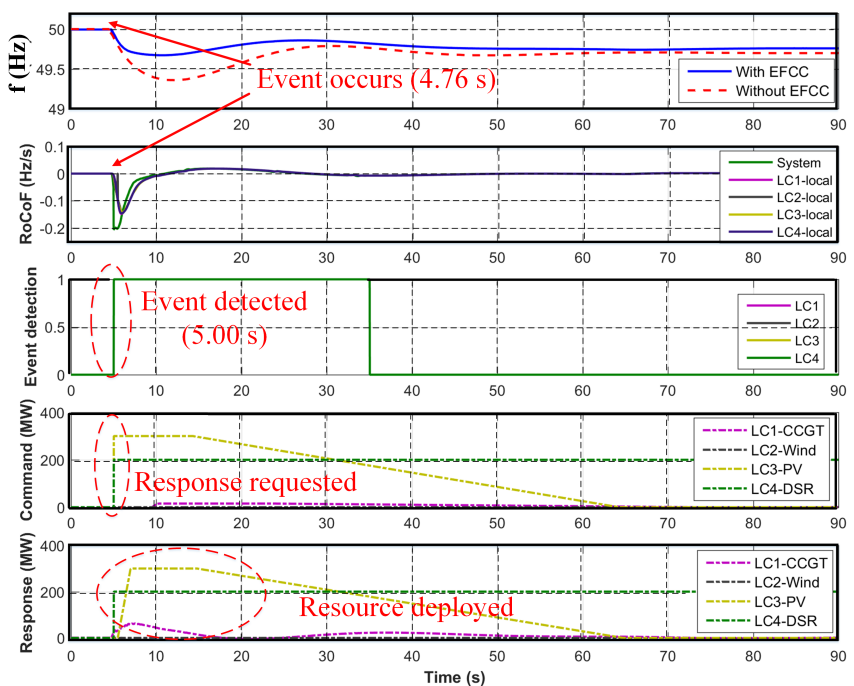

(a) Test results from Case A1 using C-HiL

Fig. 7. Test results using the C-HiL setup
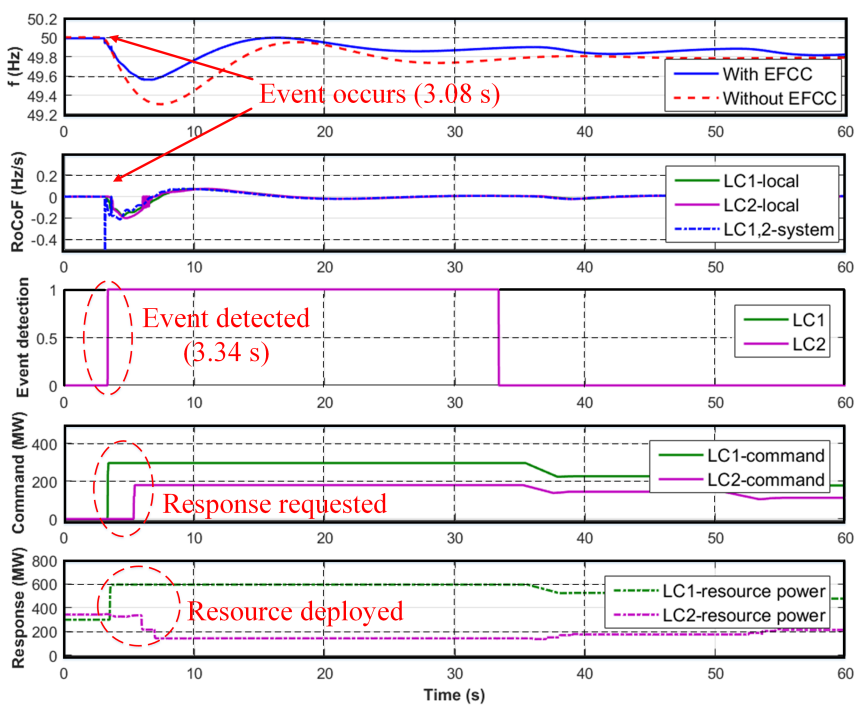

(a) Test results from Case B1 using P-HiL

Fig. 8. Test results using the P-HiL setup

frequency disturbances triggered in simulation to be accurately emulated in the $11 \mathrm{kV}$ physical network and changes in demand level at the load bank (controlled by one of the LCs) can be scaled and reflected in the simulation, acting as frequency response (more information about this setup is reported in [29]).

In this setup, the scheme has three RAs and two LCs. The simulated network model has been configured to contain three coherent regions, corresponding to the three RAs. Virtual PMU models are installed across the network, streaming real-time synchrophasor data to the three RAs. One LC (LC1) controls an energy storage resource modelled in RTDS and the other LC (LC2) controls the physical load bank at PNDC acting as demand side response. The resource information for the two LCs is provided in Table II. In addition to the PMUs installed across the network, LC2 is equipped with a physical PMU
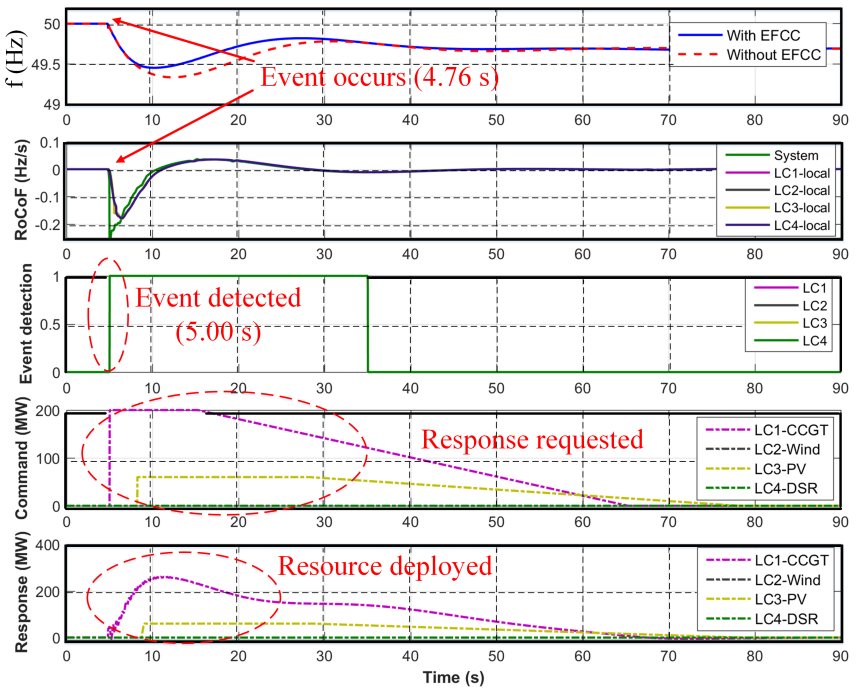

(b) Test results from Case A2 using C-HiL
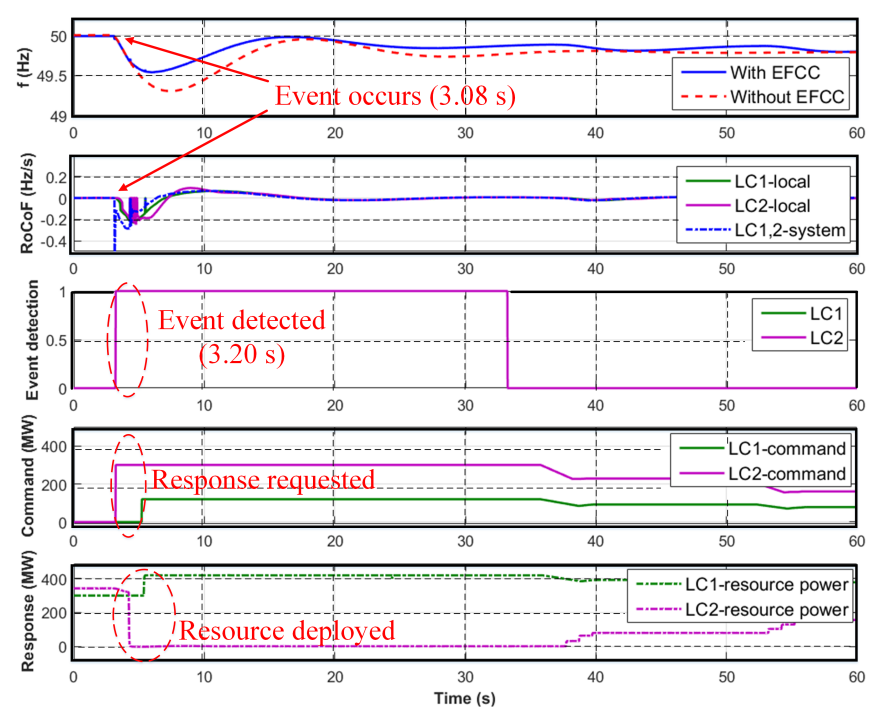

(b) Test results from Case B2 using P-HiL

installed at the $11 \mathrm{kV}$ network, while $\mathrm{LC} 1$ uses a modelled PMU in RTDS model

In the first test case using the P-HiL setup (Case B1), a $1 \mathrm{GW}$ frequency event is triggered in Region 1 , where LC1 is located. The test results are shown in Fig. 8.(a). The frequency event occurred at $3.08 \mathrm{~s}$, which was successfully detected by both LCs at $3.34 \mathrm{~s}$. LC1, which is closest to the event, requested its full $300 \mathrm{MW}$ power immediately after the event is detected, while LC2 requested $180 \mathrm{MW}$ at $5.34 \mathrm{~s}$. As discussed previously, this is because Region 1 is most severely affected by the event. This control action aims to minimise the regional variation in frequency and the angle separation. LC2 has a delay in responding to this event, which is to avoid stressing the angle separation during the event. Comparing the frequency behaviour with and without the EFCC response, it can be seen that the frequency nadir has been successfully 
TABLE I

EFCC RESOURCE INFORMATION FOR C-HIL TEST

\begin{tabular}{ccccc}
\hline Parameter & LC1 & LC2 & LC3 & LC4 \\
\hline Type & CCGT & Wind & PV & DSR \\
\hline$A$ & Yes & $\begin{array}{c}\text { Yes (case A1) } \\
\text { No (case A2) }\end{array}$ & Yes & Yes \\
\hline$P^{+}$ & $200 \mathrm{MW}$ & $300 \mathrm{MW}$ & $300 \mathrm{MW}$ & $200 \mathrm{MW}$ \\
\hline$P^{-}$ & $300 \mathrm{MW}$ & $200 \mathrm{MW}$ & $100 \mathrm{MW}$ & $0 \mathrm{MW}$ \\
\hline$T_{D}^{+}$ & $300 \mathrm{~ms}$ & $100 \mathrm{~ms}$ & $100 \mathrm{~ms}$ & N/A \\
\hline$T_{D}^{-}$ & $300 \mathrm{~ms}$ & $100 \mathrm{~ms}$ & $100 \mathrm{~ms}$ & N/A \\
\hline$d P^{+} / d t$ & $300 \mathrm{MW} / \mathrm{s}$ & $1000 \mathrm{MW} / \mathrm{s}$ & $1000 \mathrm{MW} / \mathrm{s}$ & N/A \\
\hline$d P^{-} / d t$ & $300 \mathrm{MW} / \mathrm{s}$ & $1000 \mathrm{MW} / \mathrm{s}$ & $1000 \mathrm{MW} / \mathrm{s}$ & N/A \\
\hline$T_{P}^{+}$ & $10 \mathrm{~s}$ & $10 \mathrm{~s}$ & $10 \mathrm{~s}$ & $120 \mathrm{~s}$ \\
\hline$T_{P}^{-}$ & $10 \mathrm{~s}$ & $10 \mathrm{~s}$ & $10 \mathrm{~s}$ & N/A \\
\hline Region & 1 & 1 & 2 & 2 \\
\hline Ranking & 2 & 1 & 1 & 2 \\
\hline
\end{tabular}

TABLE II

EFCC RESOURCE INFORMATION FOR P-HIL TEST

\begin{tabular}{ccc}
\hline Parameter & LC1 & LC2 \\
\hline Type & Energy storage (virtual) & DSR (physical load) \\
\hline$A$ & Yes & Yes \\
\hline$P^{+}$ & $300 \mathrm{MW}$ & $300 \mathrm{MW}$ \\
\hline$P^{-}$ & $300 \mathrm{MW}$ & $300 \mathrm{MW}$ \\
\hline$T_{D}^{+}$ & $100 \mathrm{~ms}$ & $500 \mathrm{~ms}$ \\
\hline$T_{D}^{-}$ & $100 \mathrm{~ms}$ & $500 \mathrm{~ms}$ \\
\hline$d P^{+} / d t$ & $1000 \mathrm{MW} / \mathrm{s}$ & $1000 \mathrm{MW} / \mathrm{s}$ \\
\hline$d P^{-} / d t$ & $1000 \mathrm{MW} / \mathrm{s}$ & $1000 \mathrm{MW} / \mathrm{s}$ \\
\hline$T_{P}^{+}$ & $80 \mathrm{~s}$ & $80 \mathrm{~s}$ \\
\hline$T_{P}^{-}$ & $80 \mathrm{~s}$ & $80 \mathrm{~s}$ \\
\hline Region & 1 & 1 \\
\hline Ranking & 1 & 1 \\
\hline
\end{tabular}
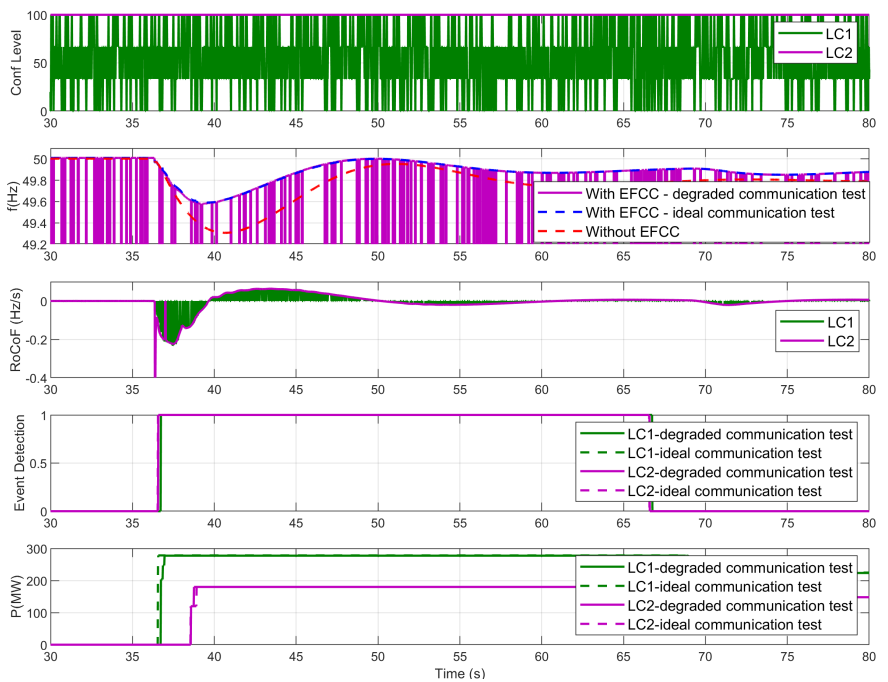

Fig. 9. Impact of jitter on the operation of the EFCC controllers

raised from $49.30 \mathrm{~Hz}$ to $49.57 \mathrm{~Hz}$. The last plot shows the load bank changed its output in a number of steps. This is due to the nature of the load bank's internal controller, which only updates the load level approximately every $1 \mathrm{~s}$.

For the second test using P-HiL (Case B2), a $1 \mathrm{GW}$ loss of infeed frequency disturbance was trigged in Region 3, where LC2 is located. The test results are presented in Fig. 8.(b). It can be seen that the event occurred at approximately $3.08 \mathrm{~s}$. In this case LC2 requested its full power (300 MW) immediately after the event is detected at $3.20 \mathrm{~s}$, while LC1 has a delay in responding to this event at $5.20 \mathrm{~s}$ with 120 MW power deployed. Comparing with the case without the EFCC's response, the frequency nadir has been successfully raised from $49.30 \mathrm{~Hz}$, to $49.54 \mathrm{~Hz}$.

\section{Validation of EFCC scheme with degraded communication performance}

In this work, extensive tests have been conducted to investigate the EFCC system's performance under a wide range of degraded communication conditions, e.g. loss of packets and issues associated with latency and jitter. In this case study, a high latency and jitter level (which leads to significant levels of data loss during transmission) will be emulated at the communications links between RAs and LC1 to evaluate the EFCC's performance during degraded communication conditions.

From a number of other tests conducted, it was found that the absolute maximum latency between RAs and the LCs that can be tolerated is $78 \mathrm{~ms}$. This is associated with the buffering window of the LCs, which is configured as $100 \mathrm{~ms}$. If the buffering window is increased to $200 \mathrm{~ms}$, the maximum tolerable latency will become $178 \mathrm{~ms}$. In this example, a buffering window of $100 \mathrm{~ms}$ is used. For the communication channels, a mean latency of $78 \mathrm{~ms}$ and a jitter level of 26 ms is emulated between the RAs to LC1, while there is no delay in the links between RAs and LC2 for comparison. The emulated communication condition represents the maximum tolerable latency and the maximum jitter level at this mean latency that can be mathematically emulated (any jitter greater than this value will lead to latency with negative values in the probability distribution curve, which is not physically possible). Therefore, the emulated condition for the test is indeed an extreme communication degradation scenario.

The test results from an under-frequency event are shown in Fig. 9. The first plot shows the "confidence level", which is a quantity used by the LC to evaluate the communications channel condition. If a data packet from any RA is lost or not delivered in time, the confidence level will drop by $33.33 \%$ (given that there are three RAs and thus three communication links). In this case, it can be seen that the many packets are lost due to excessive delay. When the delay is larger than the absolute tolerable value, the data will be discarded, so it is equivalent to the loss of packets. The second and third plots present the frequency and RoCoF measured with the degraded communication conditions.

It can be seen that, at this level of latency and jitter, LC1 missed packets from two RAs for the majority of the test period (confidence level dropped to $33.33 \%$ on numerous occasions). In some cases, LC1 missed data from all of the 
three RAs (confidence level dropped to $0 \%$ ). As a result, $\mathrm{LC1}$ lost wide-area visibility most frequently during the test - this is evident in the frequency and RoCoF measurement at $\mathrm{LC} 1$, which dropped frequently to 0 . However, when the frequency event occurred, the test results show that LC1 can still detect the event promptly and respond correctly to the event - very similar behaviour with the base case where the communication links were operating under ideal conditions, which is also shown in Fig. 9. This demonstrates that even with high loss of data due to the excessive communication delays and jitter, the system is still highly robust in performing its actions. This is achieved by the application of buffering windows and data interpolation techniques.

\section{Conclusions}

This paper has presented the design and validation of the EFCC scheme, which is a WAMC system capable of detecting and analyzing the regional impact of disturbances, and subsequently deploying fast and coordinated responses with consideration of the characteristics and capabilities of a range of different resources. Case studies have been presented, using both $\mathrm{C}-\mathrm{HiL}$ and P-HiL approaches to test the EFCC scheme. The test results have demonstrated that the scheme is capable of fast detection of frequency events and it can deploy FFR in a coordinated and optimized manner to enhance frequency restoration in low-inertia systems, thus providing a promising solution for the control of future power systems.

\section{REFERENCES}

[1] J. Zhu, C. Booth, G. Adam, A. Roscoe, and C. Bright, "Inertia emulation control strategy for vsc-hvdc transmission systems," IEEE Trans. on Power Systems, vol. 28, no. 2, pp. 1277-1287, May 2013

[2] National Grid ESO, "System Operability Framework 2016," Tech. Rep., 2016.

[3] P. Tielens and D. Hertem, "The relevance of inertia in power systems," Renewable and Sustainable Energy Reviews, vol. 55, pp. 999-1009, 2016.

[4] F. Milano, F. Dorfler, G. Hug, D. Hill, and G. Verbic, "Foundations and challenges of low-inertia systems," in The 20th Power Systems Computation Conference, 2018.

[5] Q. Hong, M. Nedd, S. Norris, et al., "Fast frequency response for effective frequency control in power systems with low inertia," in 14th IET International Conference on AC and DC Power Transmission, 2018.

[6] D. Wilson, S. Clark, et al., "Advances in Wide Area Monitoring and Control to address Emerging Requirements related to Inertia, Stability and Power Transfer," in CIGRE Paris Session, 2016.

[7] M. Syed, E. Guillo-Sansano, S. Blair, A. Roscoe, and G. Burt, "A novel decentralized responsibilizing primary frequency control," IEEE Trans. on Power Systems, vol. 33, no. 3, pp. 3199-3201, May 2018.

[8] P. Babahajiani, Q. Shafiee, and H. Bevrani, "Intelligent demand response contribution in frequency control of multi-area power systems," IEEE Trans. on Smart Grid, vol. 9, no. 2, pp. 1282-1291, March 2018.

[9] A. Junyent-Ferr, Y. Pipelzadeh et al., "Blending hvdc-link energy storage and offshore wind turbine inertia for fast frequency response," IEEE Trans. on Sustainable Energy, vol. 6, no. 3, pp. 1059-1066, July 2015.

[10] O. Adeuyi, M. Cheah-Mane, J. Liang, and N. Jenkins, "Fast Frequency Response From Offshore Multiterminal VSC-HVDC Schemes," IEEE Trans. on Power Delivery, vol. 32, no. 6, pp. 2442-2452, Dec 2017.

[11] E. Vrettos, C. Ziras, and G. Andersson, "Fast and reliable primary frequency reserves from refrigerators with decentralized stochastic control," IEEE Trans. on Power Systems, vol. 32, no. 4, pp. 2924-2941, July 2017.

[12] D. Chakravorty, B. Chaudhuri, and R. Hui, "Rapid frequency response from smart loads in great britain power system," IEEE Trans. on Smart Grid, vol. 8, no. 5, pp. 2160-2169, Sept 2017.

[13] D. Ochoa and S. Martinez, "Fast-frequency response provided by dfigwind turbines and its impact on the grid," IEEE Trans. on Power Systems, vol. 32, no. 5, pp. 4002-4011, Sept 2017.
[14] D. Yang, J. Kim, Y. Kang, E. Muljadi, et al., "Temporary frequency support of a dfig for high wind power penetration," IEEE Trans. on Power Systems, vol. 33, no. 3, pp. 3428-3437, May 2018.

[15] A. Hoke, M. Shirazi, S. Chakraborty, E. Muljadi, and D. Maksimovic, "Rapid active power control of photovoltaic systems for grid frequency support," IEEE Journal of Emerging and Selected Topics in Power Electronics, vol. 5, no. 3, pp. 1154-1163, Sept 2017.

[16] D. Gautam, L. Goel, R. Ayyanar, V. Vittal, and T. Harbour, "Control strategy to mitigate the impact of reduced inertia due to doubly fed induction generators on large power systems," IEEE Trans. on Power Systems, vol. 26, no. 1, pp. 214-224, Feb 2011.

[17] B. Craciun, T. Kerekes, et al., "Frequency support functions in large pv power plants with active power reserves," IEEE Journal of Emerging and Selected Topics in Power Electronics, vol. 2, no. 4, pp. 849-858, Dec 2014.

[18] H. Xin, Y. Liu, Z. Wang, D. Gan, et al. , "A new frequency regulation strategy for photovoltaic systems without energy storage," IEEE Trans. on Sustainable Energy, vol. 4, no. 4, pp. 985-993, Oct 2013.

[19] T. Xu, W. Jang, and T. Overbye, "Commitment of fast-responding storage devices to mimic inertia for the enhancement of primary frequency response," IEEE Trans. on Power Systems, vol. 33, no. 2, pp. 1219-1230, March 2018.

[20] F. Teng, V. Trovato, and G. Strbac, "Stochastic scheduling with inertiadependent fast frequency response requirements," IEEE Trans. on Power Systems, vol. 31, no. 2, pp. 1557-1566, March 2016.

[21] F. Wilches-Bernal, R. Concepcion, J. Neely, R. Byrne, and A. Ellis, "Communication enabled-fast acting imbalance reserve (ce-fair)," IEEE Trans. on Power Systems, vol. 33, no. 1, pp. 1101-1103, Jan 2018.

[22] National Grid ESO, "Technical Report on the Events of 9 August 2019," Tech. Rep., 2019.

[23] D. Wilson, O. Bagleybter, S. Norris and K. Maleka, "Control of an electrical power network," Patent WO2 $016174476 \mathrm{~A} 3$, Nov 03, 2016. [Online]. Available: https://patents.google.com/patent/WO2016174476A3/en

[24] H. You, V. Vittal, and X. Wang, "Slow coherency-based islanding," IEEE Trans. on Power Systems, vol. 19, no. 1, pp. 483-491, Feb 2004.

[25] "IEEE Std C37.118.1-2011 - IEEE Standard for Synchrophasor Measurements for Power Systems," 2011.

[26] P. Kundur, Power System Stability and Control. McGraw-Hill, 1993.

[27] DERlab, "European White Book on Real-Time Powerhardware-in-theLoop Testing," Tech. Rep., 2011.

[28] A. Vijay, S. Doolla, and M. Chandorkar, "Real-time testing approaches for microgrids," IEEE Journal of Emerging and Selected Topics in Power Electronics, vol. 5, no. 3, pp. 1356-1376, Sept 2017.

[29] Q. Hong, I. Abdulhadi, et al., "Application of a MW-scale motorgenerator set to establish P-HiL capability," in IEEE ISGT Europe, 2017.

[30] GE Grid Solutions, "Control Platform Specification Summary," Tech. Rep., 2015.

[31] P. M. Ashton, C. S. Saunders, G. A. Taylor, A. M. Carter, and M. E. Bradley, "Inertia estimation of the gb power system using synchrophasor measurements," IEEE Transactions on Power Systems, vol. 30, no. 2, pp. 701-709, March 2015.

[32] Power System Dynamic Performance Committee, IEEE Power and Energy Society, "Dynamic Models for Turbine-Governors in Power System Studies," Jan 2013.

[33] F. Terreros, "Develop of the Dynamic Model of GB for Transient Stability in RSCAD," Master's thesis, Delft University of Technology, 2017.

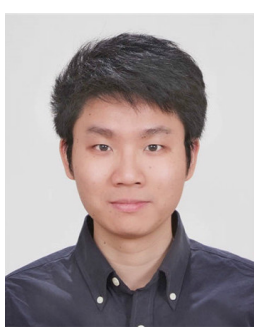

Qiteng Hong (S'11-M'15) is currently a Research Fellow at the University of Strathclyde, Glasgow, U.K. His main research interest is on power system protection and control in future networks with low inertia, resulting from high penetration of renewable generation. He received his B.Eng. (Hons) and Ph.D. degree in Electronic and Electrical Engineering in 2011 and 2015 respectively, both from the University of Strathclyde. He is a Regular Member of the CIGRE Working Group B5.50, IEEE Working Group P2004, and the Technical Lead at the CIGRE UK 


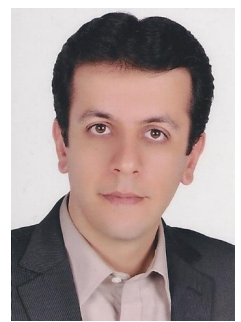

Mazaher Karimi (M'11) received the B.Eng. degree from Islamic Azad University, Iran, in 2002 and the M.Eng. degree in electrical engineering and the Ph.D. degree from the University of Malaya, Malaysia, in 2011 and 2013, respectively. Currently, he is an Assistant Professor with Department of Electrical Engineering, Faculty of Basic Sciences and Engineering, Gonbad Kavous University, Iran. His main research interest is in distributed generation and electric power system stability and frequency control.

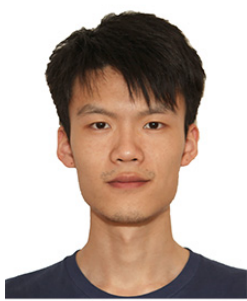

Mingyu Sun (S'15) received the B.Eng. degree in Electrical Engineering from Shandong University, Jinan, China in 2013 and the M.Sc. degree in Electrical Power System Engineering from the University of Manchester, Manchester, UK in 2014. $\mathrm{He}$ is currently working toward the Ph.D. degree in Electrical Engineering at the University of Manchester, Manchester, UK. His research interests include application of power electronics in power systems, and wide-area monitoring, protection and control. He has been also with National Grid Electricity Transmission as an innovation engineer since 2019.

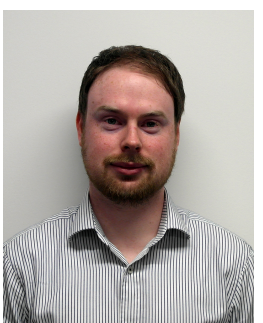

Seán Norris is a Senior Power Systems Engineer with GE Renewables based in Edinburgh, UK. He received his B.Eng from University College Cork, Ireland in 2009 and $\mathrm{PhD}$ from Durham University, UK in 2014. Seán's areas of expertise include wide-area monitoring and control of power systems, control system application design, power system stability and operation under emergency conditions and fast frequency control. His current projects focus on the design and implementation of innovative fast wide-area control schemes to enhance grid stability.

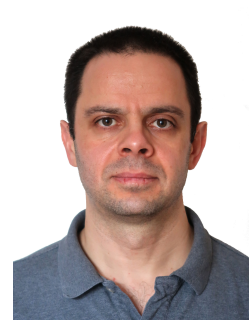

Oleg Bagleybter is currently working as a Director - Data \& Analytics at GE and is responsible for WAMS product portfolio within the GE Grid Solution business. His focus is on developing and deploying innovative applications and solutions utilising Wide Area measurements in Transmission and Distribution Grids. He received the Diploma in Electrical Engineering and Ph.D. degree from the Irkutsk Technical University in 1999 and 2006 respectively. $\mathrm{He}$ has worked in the past as a Protection and Control Engineer for one of the Russian utilities and a Product Manager for transmission protection relays for GE Grid Solutions.

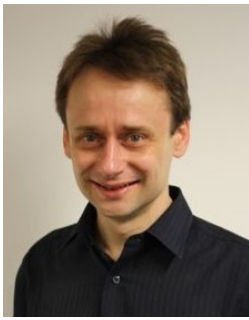

Douglas Wilson is Principle Analytics Engineer with GE, specializing in power system dynamics and stability, synchrophasor measurements and wide area control, with a current focus on low inertia system control. He graduated with B.Eng and $\mathrm{PhD}$ from the University of Edinburgh and MSc from the University of Manchester, and was CTO for Psymetrix Ltd before its acquisition by Alstom and then GE. He is an IEEE Senior Member and Fellow in GE's Technical Expert Program. .

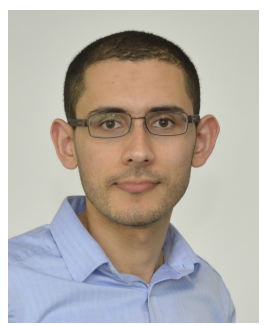

Ibrahim F. Abdulhadi (S'08-M'11) received the M.Eng. and Ph.D. degrees in electronic and electrical engineering from the University of Strathclyde, U.K., in 2007 and 2013. He is currently an Research and Development Engineer within the university's Power Networks Demonstration Centre. He has experience working for U.K. distribution and transmission network operators. His research interests include power system protection, real-time power system simulation, hardware in the loop testing and communications applications in smart grids.

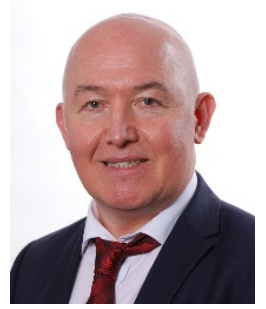

Vladimir Terzija (M'95-SM'00-F'16) received the Dipl-Ing., M.Sc., and Ph.D. degrees in electrical engineering from the University of Belgrade in 1988 , 1993, and 1997, respectively. He is the EPSRC Chair Professor in Power System Engineering with the School of Electrical and Electronic Engineering, The University of Manchester. His current research interests include smart grid applications; wide-area monitoring, protection, and control; multi-energy systems; switchgear and transient processes; ICT, data analytics and digital signal processing applications in power systems. Prof. Terzija is Editor in Chief of the International Journal of Electrical Power and Energy Systems, Alexander von Humboldt Fellow, as well as a DAAD and Taishan Scholar. He is the National Thousand Talents Distinguished Professor at Shandong University, China.

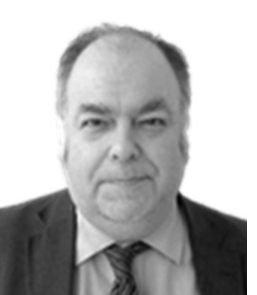

Ben Marshall joined National Grid in 1996. Over the last 20 years, he has analysed and developed the operation and design of the GB network, including delivery of early reactive power market and frequency service contract assessments, the connection design and wider network reinforcement to accommodate conventional power stations, the first transmission connected wind, and the first new HVDC connections to the system; contributing to the development of power system assessment processes which underpin this. He has input to several technical and commercial code and security standard reviews impacting both Transmission and distribution networks, and across networks development strategies, for example those now active within central London and across the South East of England. In his current role as system performance technical specialist within the GB System Operator, he provides support and analysis across areas of System Operability, discussed further in National Grids System Operability Framework publication.

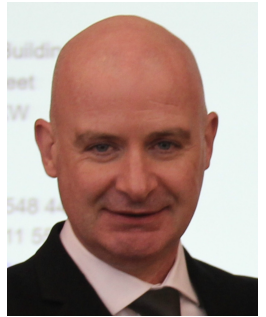

Campbell D. Booth received the B.Eng. and Ph.D. degrees in Electrical and Electronic Engineering from the University of Strathclyde, Glasgow, U.K., in 1991 and 1996, respectively. He is currently a Professor and Head of the Department for Electronic and Electrical Engineering, University of Strathclyde. His research interests include power system protection; plant condition monitoring and intelligent asset management; applications of intelligent system techniques to power system monitoring, protection, and control; knowledge management; and 